\title{
Recent Advances in Laser Ablation Modelling for Asteroid Deflection Methods
}

\author{
Nicolas Thiry ${ }^{a}$ and Massimiliano Vasile ${ }^{b}$ \\ ${ }^{a}$ University of Strathclyde, 16 Richmond St, G1 1XQ, Glasgow, UK; \\ ${ }^{b}$ University of Strathclyde, 16 Richmond St, G1 1XQ, Glasgow, UK
}

\begin{abstract}
Over the past few years, a series of studies have demonstrated the theoretical benefits of using laser ablation in order to mitigate the threat of a potential asteroid on a collision course with earth. Compared to other slow-push mitigation strategies, laser ablation allows for a significant reduction in fuel consumption since the ablated material is used as propellant. A precise modelling of the ablation process is however difficult due to the high variability in the physical parameters encountered among the different asteroids as well as the scarcity of experimental studies available in the literature. In this paper, we derive a new thermal model to simulate the efficiency of a laser-based deflector. The useful material properties are first derived from thermochemical tables and equilibrium thermodynamic considerations. These properties are then injected in a 3D axisymetrical thermal model developed in Matlab. A temperature-dependant conduction flux is imposed on the exterior boundary condition that takes into account the balance between the incident power and the power losses due to the vaporization process across the Knudsen layer and the radiations respectively. A non-linear solver is finally used and the solution integrated over the ablation front to reconstruct the net thrust and the global mass flow. Compared to an initial 1D model, this new approach shows the importance of the parietal radiation losses in the case of a CW laser. Despite the low energy conversion efficiency, this new model still demonstrates the theoretical benefit of using lasers over more conventional low-thrust strategies.
\end{abstract}

Keywords: Asteroid deflection, Laser ablation, Vaporization, FEM

\section{INTRODUCTION}

Deflection methods are traditionally divided into two main categories depending on whether or not the modification of the orbital trajectory can be done in a quasi-instantaneous way or need to be acted over a longer period of time by slowly pushing the target away of its collision course. Impulsive techniques produce an impulsive energy transfer by exploding for example a nuclear device (nuclear interceptor) or crashing a massive spacecraft (kinetic impactor) to modify the velocity of the asteroid and thus its orbital trajectory. Slow-push methods on the other hand allow for a more controllable deflection manoeuvre by exerting a small force on the asteroid over an extended period of time. This ability to manipulate precisely the trajectory of an asteroid by contrast with the relatively crude approach offered by impulsive methods explains by itself the growing interest for these slow-push methods. Over the past years many concepts have been proposed and studied with various degrees of accuracy. Among these methods, laser ablation opens a very promising path of research. Indeed, in most cases, the efficiency of the deflection technique is eventually limited by the amount of propellant available from the beginning of the mission. For methods using laser ablation, this limitation is removed as the vaporized asteroid material is used to propel itself. Thanks to this, the mass allocation can be shifted from the propellant to the power systems. Several mission concepts using laser ablation as a deflection method have been investigated in the past by the University of Strathclyde and are worthwhile mentioning. The Light Touch concept ${ }^{1}$ was proposed to change the orbital velocity of a $4 \mathrm{~m}$ diameter, 130 ton asteroid by $1 \mathrm{~m} / \mathrm{s}$ in less than 3 years using a 453kgs spacecraft. The study was performed in cooperation with the University of Southampton, Astrium Ltd and GMV-SKY as an answer the SysNova challenge, an ESA initiative. Another approach investigated has been

Further author information: (Send correspondence to Nicolas Thiry)

Nicolas Thiry: E-mail: nicolas.thiry@strath.ac.uk

Massimiliano Vasile: E-mail: massimiliano.vasile@strath.ac.uk

Nanophotonics and Macrophotonics for Space Environments VIII, edited by Edward W. Taylor,

David A. Cardimona, Proc. of SPIE Vol. 9226, 922608 - (c) 2014 SPIE

CCC code: $0277-786 X / 14 / \$ 18 \cdot$ doi: $10.1117 / 12.2060810$

Proc. of SPIE Vol. 9226 922608-1 


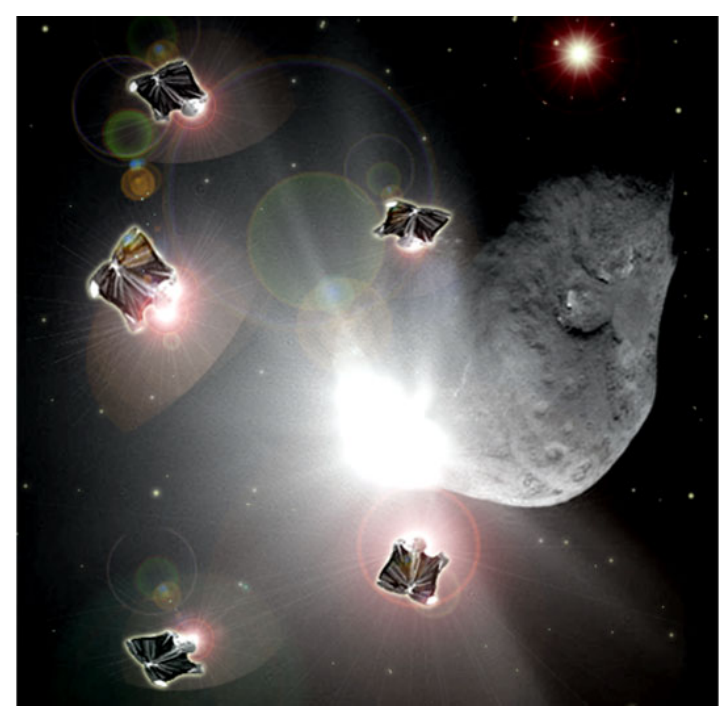

Figure 1. The Laser Bees project

the use of solar concentrators to power a laser-based deflector. ${ }^{2}$ While it may sound curious to convert solar power into another form of optical power, the higher energy density provided by the laser beam is required to reach a sufficiently high temperature to enable the vaporization process. Supported by the planetary society, the laser bees project investigates the use of a swarm of solar-pumped lasers. Flying in formation around the target asteroid, the combination of multiple beams allow to produce a higher thrust without requiring the use of a nuclear reactor. In addition, the swarm offers redundancy in the case of failure of a single spacecraft. Despite a growing interest among the scientific community, it is important to mention that none of the deflection methods, except the kinetic impactor, has had the opportunity of a real space validation. Thorough experimental investigations together with accurate numerical models are therefore essential in order to successfully validate or reject a given approach. In previous attempts to model the thermal behaviour of an asteroid during the ablation process, a 1D model was assumed as is frequently the case for laser ablation modelling ${ }^{34}$. Inevitably, such an approach neglected the power losses due to parietal conduction and radiations which can be significant whenever a continuous-wave laser is used. In order to improve our understanding, a new thermal model was developed. This new model is presented together with some results in the next section.

\section{THERMAL MODEL}

The thermal model of figure 2 relies on a finite element discretization of the heat equation coupled with a non-linear solver to handle the radiative boundary condition. Advantage is taken of the axial symmetry of the problem to reduce the number of degrees of freedom. Detailed technical information can be found in the dedicated subsection. In the first subsection, we derive the material properties of an asteroid from thermochemical tables and thermodynamic considerations.

\subsection{Physical Properties of an Asteroid}

While creating a numerical model, one should always bear in mind that in general, the quality of the outputs relies primarily on the quality of the input parameters rather than the computational power itself. We consider the case of a rocky S-type asteroid mainly composed of magnesium iron silicates. A conservative choice is to consider the vaporization of Fosterite only as it has this highest triple point among the Olivine family. Various reactions and sub-reactions can be produced during the vaporization process. A common assumption ${ }^{5}$ is that the following reaction dominates during the ablation process of Fosterite:

$$
\mathrm{Mg}_{2} \mathrm{SiO}_{4}^{(l)} \longrightarrow 2 \mathrm{MgO}^{(g)}+\mathrm{SiO}^{(g)}+\frac{1}{2} \mathrm{O}_{2}^{(g)}
$$




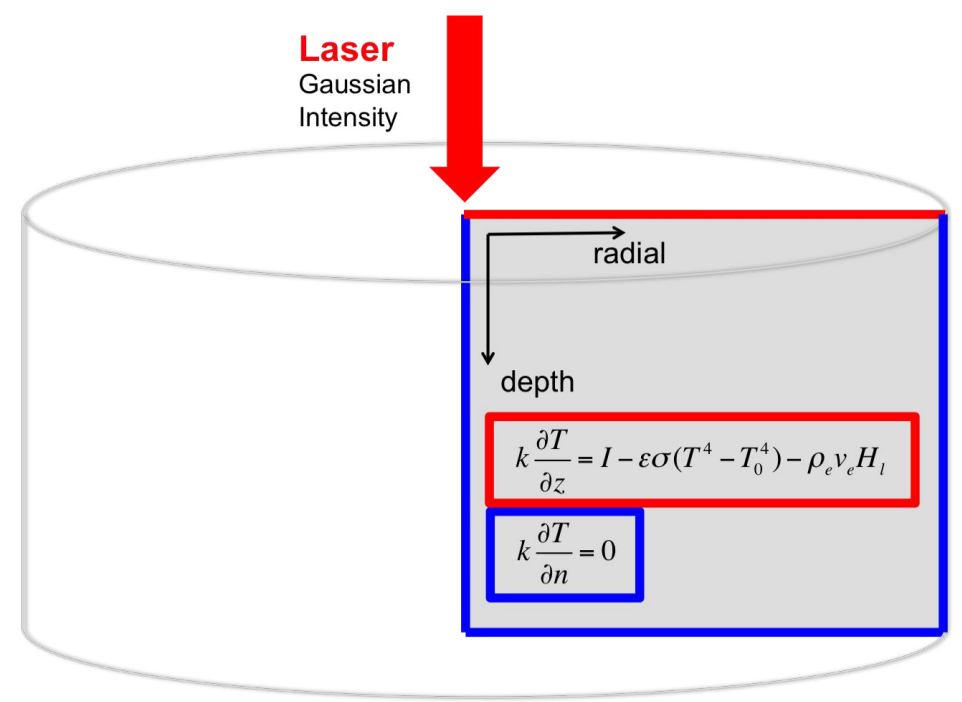

Figure 2. Axisymmetrical finite element model depicting the computational domain (slice of the asteroid) and the boundary conditions used

Following this assumption, most of the useful parameters can be directly retrieved or inferred by the data in table 1. One of the most important material properties in this respect is the vaporization enthalpy per unit mass

Table 1. Relevant properties extracted from the NIST-JANAF Thermochemical Tables ${ }^{6}$

\begin{tabular}{lllll}
\hline & $\mathrm{Mg}_{2} \mathrm{SiO}_{4}^{(l)}$ & $\mathrm{MgO}^{(g)}$ & $\mathrm{SiO}^{(g)}$ & $\mathrm{O}_{2}^{(g)}$ \\
\hline \hline$\Delta_{f}^{3500 K} \mathrm{H}^{\text {std }}\left[\mathrm{kJ} \mathrm{mol}^{-1}\right]$ & -2301.5 & -70.154 & -178.375 & 0 \\
\hline$\Delta_{f}^{3000 K} \mathrm{G}^{\text {std }}\left[\mathrm{kJ} \mathrm{mol}^{-1}\right]$ & -686.991 & -3.414 & -300.935 & 0 \\
\hline$C_{p}\left[\mathrm{~J} \mathrm{~mol}^{-1} \mathrm{~K}^{-1}\right]$ & 205 & 42 & 38 & 40 \\
\hline \hline
\end{tabular}

which can be computed by simply subtracting the formation enthalpy of the reactant to the formation enthalpy of the products as follows:

$$
\begin{aligned}
E_{v} & =\sum_{i} n_{i} \Delta_{f} H_{p_{i}}^{s t d}-\Delta_{f} H_{M g_{2} S i O_{4}}^{s t d} \\
& =0.5665 \mathrm{MJ} \cdot \mathrm{mol}^{-1}=14.163 \mathrm{MJ} \cdot \mathrm{kg}^{-1}
\end{aligned}
$$

The properties of the gas mixture formed during the ablation process can also be retrieved in a similar fashion. They are simply computed by summing the properties of the different compounds weighted by their respective molar or mass fraction in the mixture. This gives a value for the heat capacity of:

$$
c_{p}^{g}=\frac{\sum_{i} n_{i} C_{p}^{i}}{0.14}=1014.3 \mathrm{~J} \cdot \mathrm{kg}^{-1} \cdot \mathrm{K}^{-1}
$$

and a value for the gas constant of:

$$
R^{*}=\frac{\sum_{i} n_{i} R}{0.14}=207.75 \mathrm{~J} \cdot \mathrm{kg}^{-1} \cdot \mathrm{K}^{-1}
$$

The adiabatic constant can eventually be computed. The value is slightly lower than the typical 1.4 value generally assumed ${ }^{5}$ for a diatomic gas which is due to the relative convergence between the heat capacities at 
constant pressure $C_{p}$ and constant volume $C_{v}$ as temperature increases.

$$
\gamma=\frac{c_{p}^{g}}{c_{p}^{g}-R^{*}}=1.26
$$

Before modelling the ablation process, it is interesting to study the phase transition of the Forsterite compound by looking through the prism of thermodynamics. The equilibrium condition states that the phase change occurs when the chemical potentials become equal for a given state $\left\{p^{\circ}, T_{1}\right\}$.

$$
\mu^{l}\left(p^{\circ}, T_{1}\right)=\sum_{i} n_{i} \mu_{i}^{g}\left(p_{i}^{\circ}, T_{1}\right)
$$

From such an initial state, it is possible to compute the evolution of the chemical potentials with pressure and temperature. Keeping the pressure constant, the variation between two different temperature states can be derived from the so called Gibbs-Helmholtz Equation.

$$
\frac{\Delta \mu^{\circ}\left(T_{2}\right)}{T_{2}}-\frac{\Delta \mu^{\circ}\left(T_{1}\right)}{T_{1}}=\Delta H_{f}^{\circ}\left(\frac{1}{T_{2}}-\frac{1}{T_{1}}\right)
$$

If the temperature is maintained constant, the pressure dependence in the gas mixture is computed knowing that

$$
\begin{aligned}
\frac{n_{i}}{n} & =\frac{p_{i}}{p}=\frac{p_{i}^{\circ}}{p^{\circ}} \Rightarrow \frac{p_{i}}{p_{i}^{\circ}}=\frac{p}{p^{\circ}} \\
\mu_{i} & =\mu_{i}^{\circ}+R T \ln \left(\frac{p_{i}}{p_{i}^{\circ}}\right)=\mu_{i}^{\circ}+R T \ln \left(\frac{p}{p^{\circ}}\right)
\end{aligned}
$$

Since the Gibbs free energy is a state variable, we can use these two relations to compute any new state $\left\{p, T_{2}\right\}$ from the initial state $\left\{p^{\circ}, T_{1}\right\}$. Using both relations yields to the following relation that describes the new state

$$
\frac{\sum_{i} n_{i}\left(\mu_{i}^{g}\left(p_{i}, T_{2}\right)-R T_{2} \ln \left(\frac{p}{p^{\circ}}\right)\right)-\mu^{l}(p, T)}{T_{2}}=E_{v}^{\operatorname{mol}(l)}\left(\frac{1}{T_{2}}-\frac{1}{T_{1}}\right)
$$

For this state to remain an equilibrium, we must also satisfy

$$
\mu^{l}\left(p, T_{2}\right)=\sum_{i} n_{i} \mu_{i}^{g}\left(p_{i}, T_{2}\right)
$$

allowing us to suppress these terms from equation 9. In the end, we are left with equation 10 which is in fact identical to the well-known Clausius-Clapeyron formula for the gas mixture.

$$
\ln \left(\frac{p}{p^{\circ}}\right)=\frac{E_{v}^{\operatorname{mol}(l)}}{R \sum_{i} n_{i}}\left(\frac{1}{T_{1}}-\frac{1}{T_{2}}\right)=\frac{E_{v}}{R^{*}}\left(\frac{1}{T_{1}}-\frac{1}{T_{2}}\right)
$$

The reference state can eventually be computed from the formation free Gibbs enthalpy of the different species provided at standard pressure conditions and $3000 \mathrm{~K}$ in table 1.

$$
\begin{aligned}
\Delta G_{\text {mol }(g)}^{\text {std }} & =\sum_{i} \frac{n_{i}}{n}\left(\Delta_{f} G_{i}^{s t d}+R T \ln \left(\frac{n_{i}}{n}\right)\right)-\frac{\Delta_{f} G_{f o s t}^{s t d}}{n} \\
\Delta G_{\text {mol }(g)}^{\circ} & =\Delta G_{\text {mol }(g)}^{s t d}+R T \ln \left(\frac{p^{\circ}}{10^{5}}\right)=0 \\
p^{\circ} & =10^{5} \exp \left(\frac{-\Delta G_{\text {mol }}^{s t d}}{R T}\right)=4448.9 P a
\end{aligned}
$$

At $3000 \mathrm{~K}$, we find a pressure of 4448.9 Pascals. Knowing this calibration point, the whole curve can now be computed and the result is represented on figure 3 .

Note that this curve is consistent with the results of previous studies. ${ }^{7}$ 


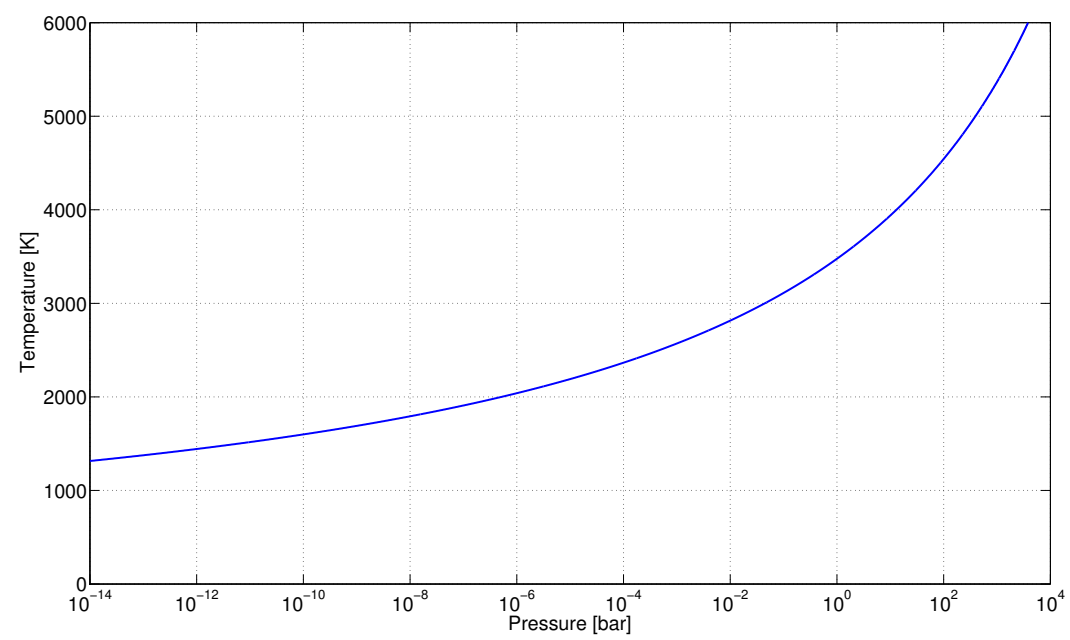

Figure 3. Calculated equilibrium vapor pressure of the system $\mathrm{Mg}_{2} \mathrm{SiO}_{4}^{(l)} \leftrightarrow 2 \mathrm{MgO}(g)+\mathrm{SiO}^{(g)}+\frac{1}{2} \mathrm{O}_{2}^{(g)}$

\subsection{Finite Element Model}

The thermal model was implemented in Matlab using the PDE toolbox to solve the heat equation 12 in cylindrical coordinates. In this equation, s represents a volumic source term which is equal to zero if we assume that the asteroid material is opaque and the power from the laser beam is absorbed at the surface of the ablation spot. However, in the case of a partially transparent material, this term will account for the absorption of the beam intensity through the depth of the material. In the discussion part of this paper, we show the critical importance of this effect which is poorly characterized in the current literature.

$$
\nabla \cdot(k \nabla T)+s=0
$$

A typical mesh can be seen on figure 4. The mesh criterion and geometry size is automatically adapted in function of the user-defined input power and diameter of the laser beam. These were initially adjusted by following a thorough sensitivity study to ensure they do not compromise the quality of the results. The mass flow during the vaporization process is a result of the thermodynamical non-equilibrium at the interface. As they vaporize, the molecules acquire an average translational velocity component. This is due to mass conservation and the combination of an abrupt change of density and the relative motion of the interface. While this interface motion is typically on the order of a few tenth of millimetres per second, the density is divided by several millions through the passage of the interface. The finite layer through which this velocity is acquired is called the Knudsen layer and can be treated as a gas-dynamic discontinuity. The jump conditions have been investigated by Knight ${ }^{8}$ and are given in equation 13 :

$$
\begin{aligned}
m & =\sqrt{\frac{\gamma}{2}} M_{e}=\frac{v_{e}}{\sqrt{2 R^{*} T_{e}}} \\
\frac{T_{e}}{T_{s}} & =\left[\sqrt{1+\pi\left(\frac{\gamma-1}{\gamma+1} \frac{m}{2}\right)^{2}}-\sqrt{\pi} \frac{\gamma-1}{\gamma+1} \frac{m}{2}\right]^{2} \\
\frac{\rho_{e}}{\rho_{s}} & =\sqrt{\frac{T_{s}}{T_{e}}}\left[\left(m^{2}+\frac{1}{2}\right) e^{m^{2}} \operatorname{erfc}(m)-\frac{m}{\sqrt{p i}}\right]+\frac{1}{2} \frac{T_{s}}{T_{e}}\left[1-\sqrt{\pi} m e^{m^{2}} \operatorname{erfc}(m)\right]
\end{aligned}
$$

In this expression, $\mathrm{M}_{e}$ represents the local Mach number on the edge of the Knudsen layer and is dependant on the pressure environment downstream. In vacuum, $\mathrm{M}_{e}$ equates 1 as the flow reaches the sonic limit. The mass flow can thus be computed from equation 13 once the temperature $\mathrm{T}_{s}$ of the interface is known. The conduction 


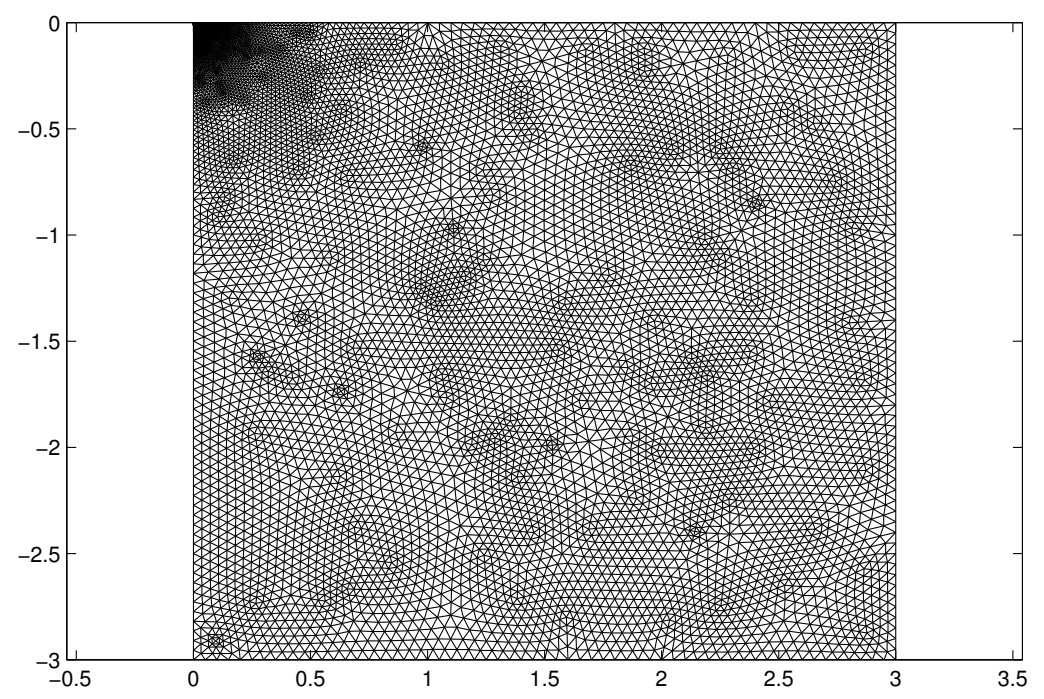

Figure 4. Mesh for the computations with local refinement in the vicinity of the ablation spot. Dimensions in meters.

far from the spot is assumed equal to zero while the conduction flux under the laser spot is imposed taking into account the incident power density and the radiation losses (proportional to the fourth power of the temperature) and the power density required to vaporize the implicit mass flow. The intensity of the laser beam is imposed with a Gaussian shape so that expression 14 naturally vanishes in the far field.

$$
q_{c}=I-\epsilon \sigma\left(T_{s}^{4}-T_{0}^{4}\right)-\rho_{e} v_{e} H_{l}
$$

The thermal problem is solved using a FEM discretization and a non-linear solver used to handle the temperaturedependant RHS term.

\subsection{Results}

Figure 5 illustrates the result obtained when one sets the optical power to $60 \mathrm{~W}$ and the diameter of the laser beam to 2 millimetres. In this case the total radiation losses integrated over the upper boundary equal $28.7 \mathrm{~W}$, which is approaching half of the available optical power. Note that about a third only of this value comes from the hot ablation spot while the rest is dissipated by the cooler but vaster neighbouring area. The net thrust is equal to $2.1 \mathrm{mN}$ in this case and is computed by numerically integrating the results over the ablation spot:

$$
\begin{aligned}
F & =\int_{\text {spot }} p_{e}+\rho_{e} v_{e}^{2} d S \\
& =\int_{\text {spot }}(1+\gamma) p_{e} d S
\end{aligned}
$$

As in a rocket exhaust, the contribution to the thrust is in fact made of two contributions: one due to the escape velocity $(806 \mathrm{~m} / \mathrm{s}$ in average for this case) and the other one due to the pressure of the exhaust gas itself pushing on the asteroid. Note that it is easy to verify that the later expansion in vacuum cannot generate any additional thrust.

Repeating the computations for different input power and beam diameter, it is possible to observe the evolution of the momentum coupling coefficient representing the amount of thrust generated per each Watt of optical power spent in the process. It appears that a narrower beam is beneficial for the efficiency and that the momentum coupling coefficient converges to a value of $80 \mu \mathrm{N} / \mathrm{W}$ as the level of power increases. 

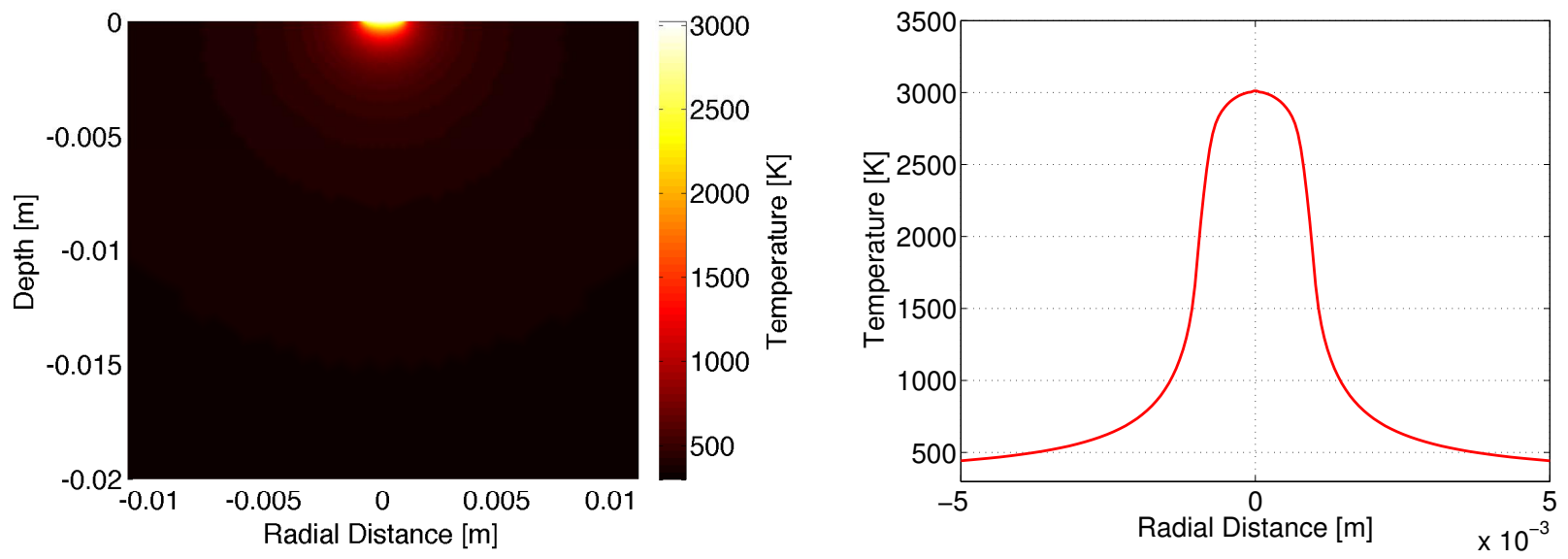

Figure 5. Temperature map inside of the domain (left) and profile (right) on the upper surface boundary. Optical power of $60 \mathrm{~W}$ and spot diameter of $2 \mathrm{~mm}$.

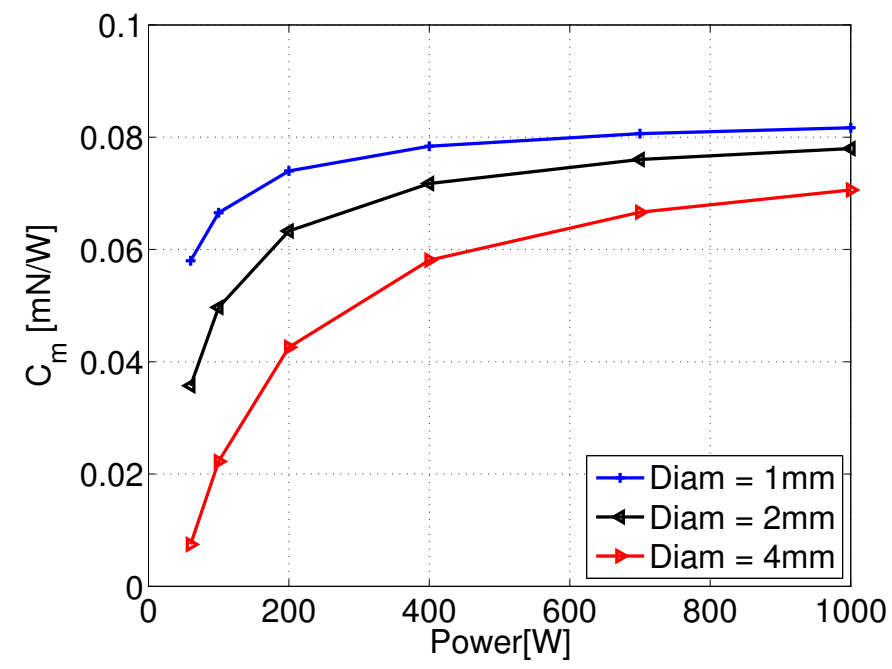

Figure 6. Momentum coupling coefficient in function of the optical power for different spot diameters 


\section{EXPERIMENTAL TECHNIQUES}

The initial objectives of the experiments are to verify if the temperature observed under the laser spot matches with the numerical predictions. According to the theoretical model developed earlier, it appears indeed that this temperature is the main driver of the different energy losses and also the key factor in establishing the vaporization rate. This section discusses the approach initially considered and also introduces an inexpensive strategy that could be used in complement and confirm the validity of the spectral temperature measurements.

\subsection{Equipment}

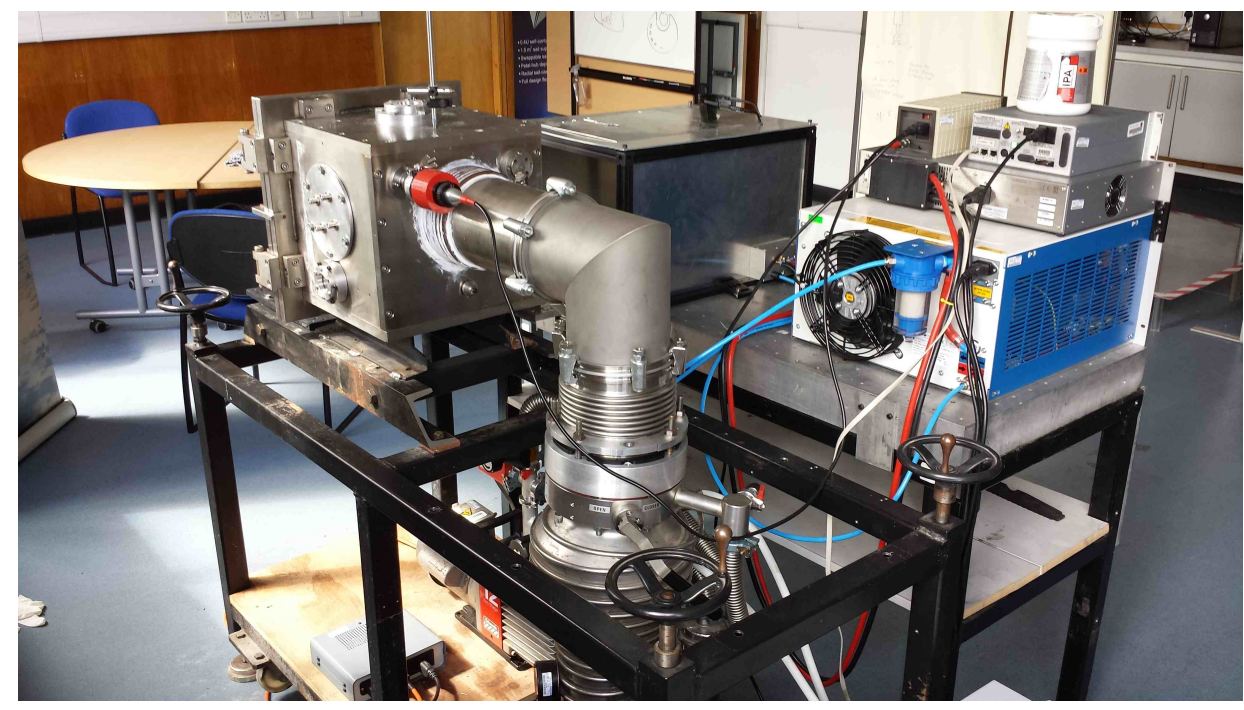

Figure 7. Laboratory Equipment

The laboratory equipment was assembled during Dr. Alison Gibbings' $\mathrm{PhD}$ thesis ${ }^{9}$ as part of a collaboration between the University of Strathclyde and the University of Glasgow and is currently located in the James Watt building. The equipment is visible on figure 7 in which the vacuum chamber and the laser equipment can be seen. A pressure of the order of a milli-pascal can be reached using a vacuum pump that comprises two stages: a rotatory pump used to remove the majority of the air molecule in the test chamber and a diffusion pump using heated oil to capture the remaining air molecules. A continuous-wave fiber-coupled diode laser is available with a maximum optical power of $90 \mathrm{~W}$ and operating in the near-infrared range $(808 \mathrm{~nm})$.

\subsection{Methodology}

A non-intrusive approach must be considered due to the high temperature levels predicted. Assuming the asteroid sample approaches the ideal behaviour of a black body, an indirect temperature measurement can be performed by looking the spectrum of the light emitted during the ablation process. Curves on figure 8 can be computed using the Plank law relating the spectral radiance to the wavelength:

$$
S(\lambda)=\frac{2 \pi c^{2} h}{\lambda^{5}} \frac{1}{e^{\frac{h c}{\lambda k T}}-1}
$$

The peak wavelength can easily be computed in function of the temperature from equation 16 .

$$
\lambda_{\text {peak }}=\frac{2.898}{1000 T}
$$

Therefore, it appears that the higher the temperature is the more its spectrum will be shifted towards the higher frequency range. A logical approach consists thus in using a spectrometer to record the emission spectrum and try to match the peak observed with the appropriate temperature using the Wien displacement law in equation 

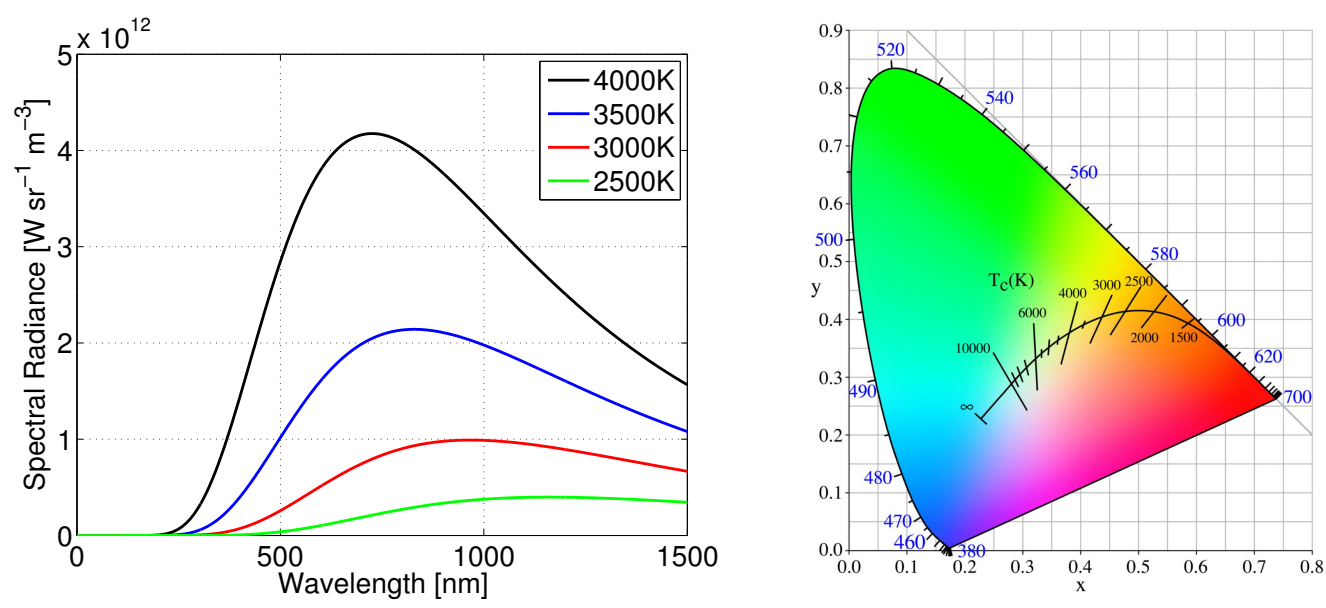

Figure 8. Spectral radiance of a black body (left) and the CIE 1931 color space and the planckian locus(right)

17. This technique was used in previous experiments but it leads to results that can be difficult to analyse in the case the sensitivity of the instrument in function of the wavelength is not a flat curve. We show here an alternative technique that can be used in complement of the spectrometer analysis and relies on the color temperature. ${ }^{10}$ The idea is just to consider a picture taken with a normal camera under the illumination provided by the natural emission of the ablation spot. Indeed each pixel of such a picture carries a temperature information encoded in its RGB combination. From equation 16, we see that a warmer source will appear as containing more blue colors while a colder source will appear to contain more red colors. Somehow, the RGB information also needs to be processed in order to remove the brightness information which is irrelevant in the temperature determination. Knowing the RGB value, a simple matrix transformation allows to compute the XYZ tristimulus values.

$$
\left(\begin{array}{l}
X \\
Y \\
Z
\end{array}\right)=\left(\begin{array}{ccc}
2.768892 & 1.751748 & 1.1302 \\
1 & 4.5907 & 0.0601 \\
0 & 0.056508 & 5.594292
\end{array}\right)\left(\begin{array}{l}
R \\
G \\
B
\end{array}\right)
$$

The proportion of each monochromatic component can then be computed by normalizing each tristimulus value by the luminance:

$$
\begin{aligned}
& x=\frac{X}{X+Y+Z} \\
& y=\frac{Y}{X+Y+Z}
\end{aligned}
$$

Last but not least, a correlated color temperature can be computed knowing the chromaticity value.

$$
\begin{aligned}
n & =\frac{x-0.3320}{y-0.1858} \\
C C T & =-449 n^{3}+3525 n^{2}-6823.3 n+5520.33
\end{aligned}
$$

As an example, figure 9 shows the application of the color technique to an image of the plume taken during a previous ablation experiment performed by Gibbings et al. Unfortunately, we see that in this case, the technique performs poorly as a majority of the pixels in the vicinity of the plume have a saturation value and thus have a corrupted temperature information embedded. Still it is remarkable to see that despite this, a large number of pixels are aligned in a range between $3000 \mathrm{~K}$ and $3500 \mathrm{~K}$ which is close to the range expected by the numerical model. In future experiment, a neutral white reflecting surface will be used to diffuse the light emitted from the ablation spot during the experiment. Recording images from this surface will allow to work around the brightness issue and also allow to ensure that the color recorded has not been corrupted by its reflection on the surfaces of the chamber. For comparison, figure 10 shows the same technique applied to the picture of a white 

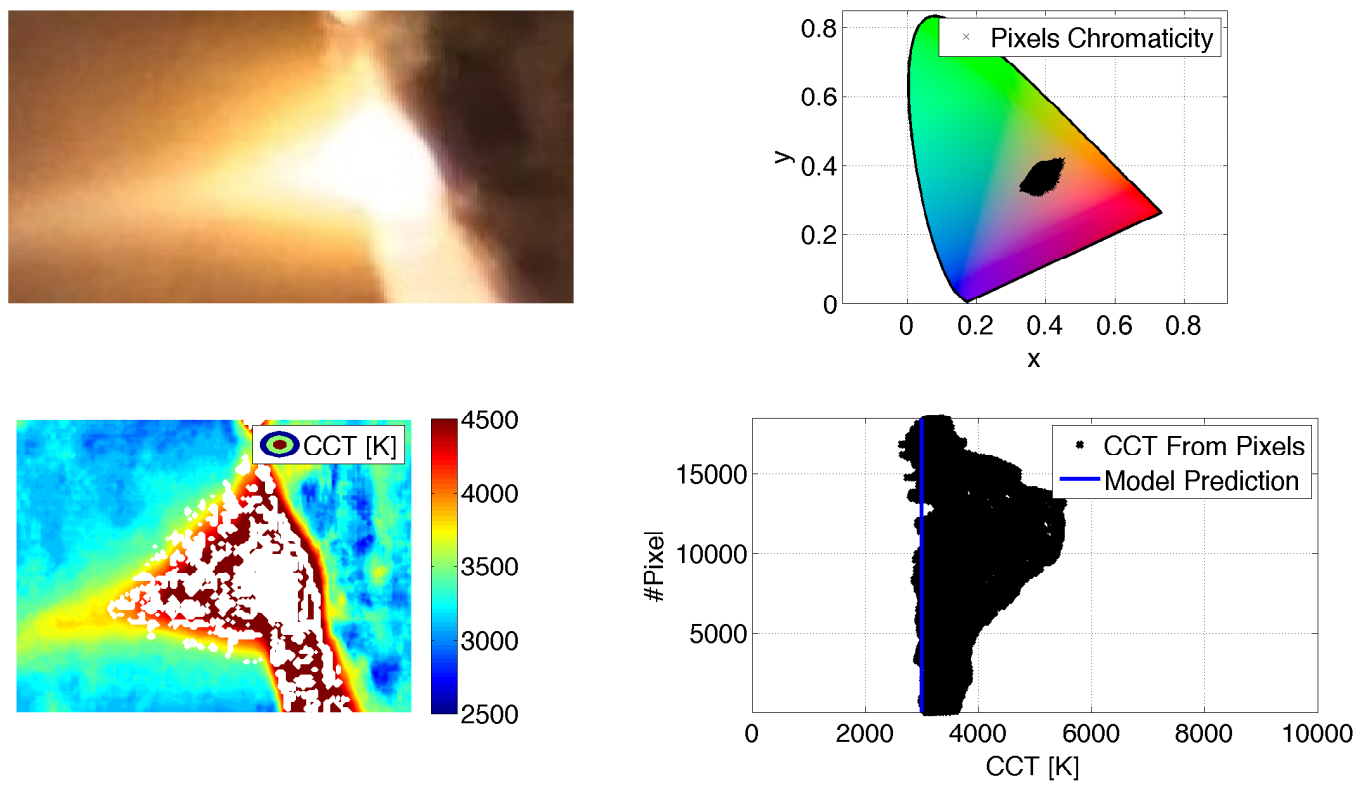

Figure 9. Application of the color technique on a picture of the plume taking during a past experiment

wall taken during a sunny day in Glasgow. In this case, we see that the pixels have a more uniform correlated color temperature. Some discrepancies are still visible due to the shadowed area and the impurities on the wall. The temperature is consistent with the observable temperature of the sun which is slightly lower than the actual temperature and is due to the high latitude and the transmission through the atmosphere of the Earth. 

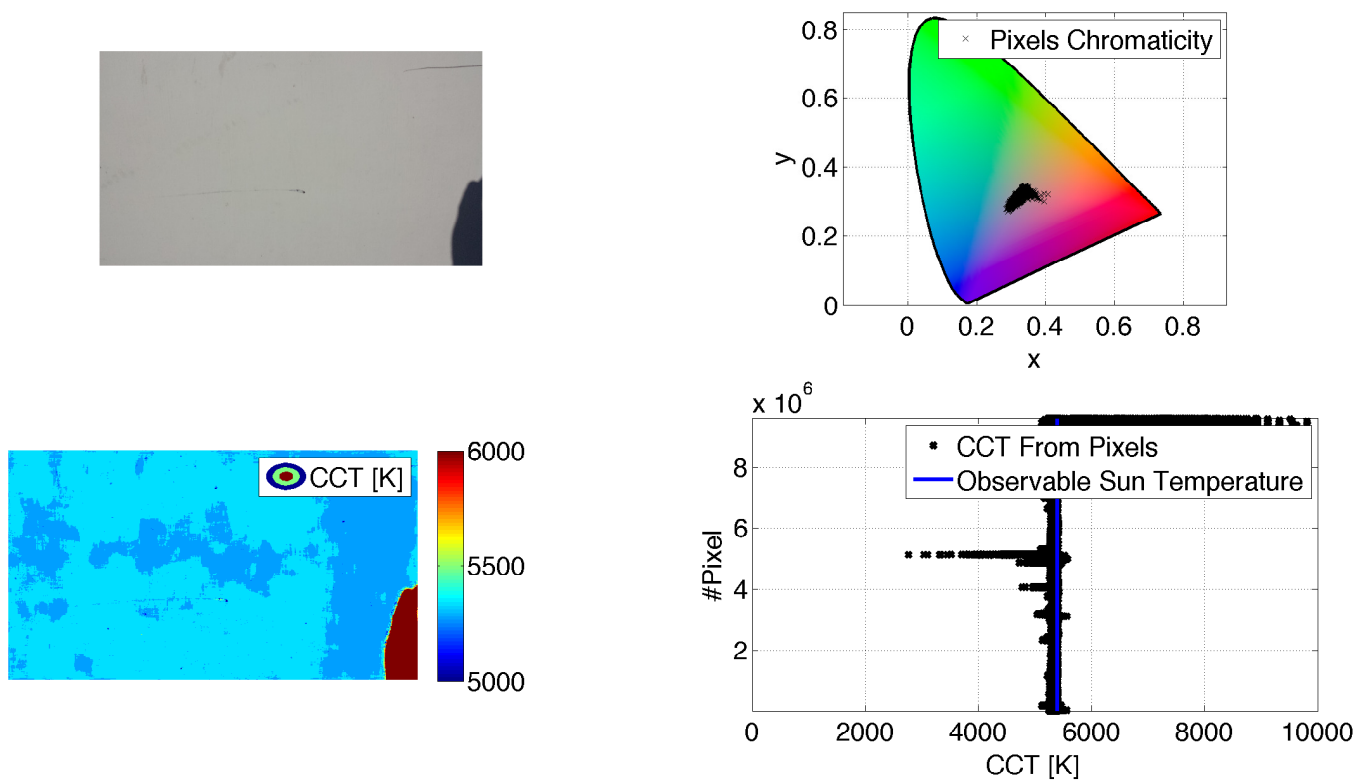

Figure 10. Application of the color technique on a picture of a white wall taken during a sunny day around noon in Glasgow 


\section{DISCUSSION}

The new model indicates that the power losses due to radiation can be important when the 3D effects are considered. For the case shown, about two-third of the radiation losses are due to the hot surface in the vicinity of the ablation spot while a third only of the losses are caused by the ablation spot itself. The heat released from the parietal radiations is in fact coming from the lateral conduction under the ablation spot. Despite this, we see that the momentum coupling predicted by the model is still considerable. For high power lasers, a value of $80 \mu \mathrm{N} / \mathrm{W}$ is expected. This does not take into account the efficiency of the energy conversion to the optical power of the laser but this shows that, in theory, a deflection method based on laser ablation can reach an efficiency comparable to the efficiency of low-thrust methods. Of course these considerations will need to be moderated by future experimental work. In practice, note that the size of the domain presented in figure 4 can be reduced to match with the finite size of the test sample used during the experiments. This will require also to modify the boundary conditions in the far field to include the radiation losses also there. Another parameter which, at our knowledge, has not been assessed in the scientific literature is the effect of a hypothetical partial transparency of the asteroid material at the wavelength used by the laser system. This effect can presently be incorporated in our model but requires the knowledge of the optical density of the ablated material. Depending on the value of the optical density, the power of the laser beam is absorbed over a certain thickness of material which is typically very small for an optically thick material but may become non-negligible for a partially transparent material. In other words, for an optically thick material, the result will be equivalent as considering that the entire radiation is absorbed on the surface of the asteroid material (which means mathematically that we consider the radiation in the boundary condition) whereas in the other case, the radiation will act as a source term in the heat equation ${ }^{11}$ - As an illustration, figure 11 shows the temperature distribution in the solid in the case the optical depth is significant compared to the size of the ablation spot. In this case, the temperature of the spot drops significantly as the heat is distributed more evenly under the skin of the material. Due to this, no significant thrust can be generated through the ablation process and almost all of the power is lost in radiations.
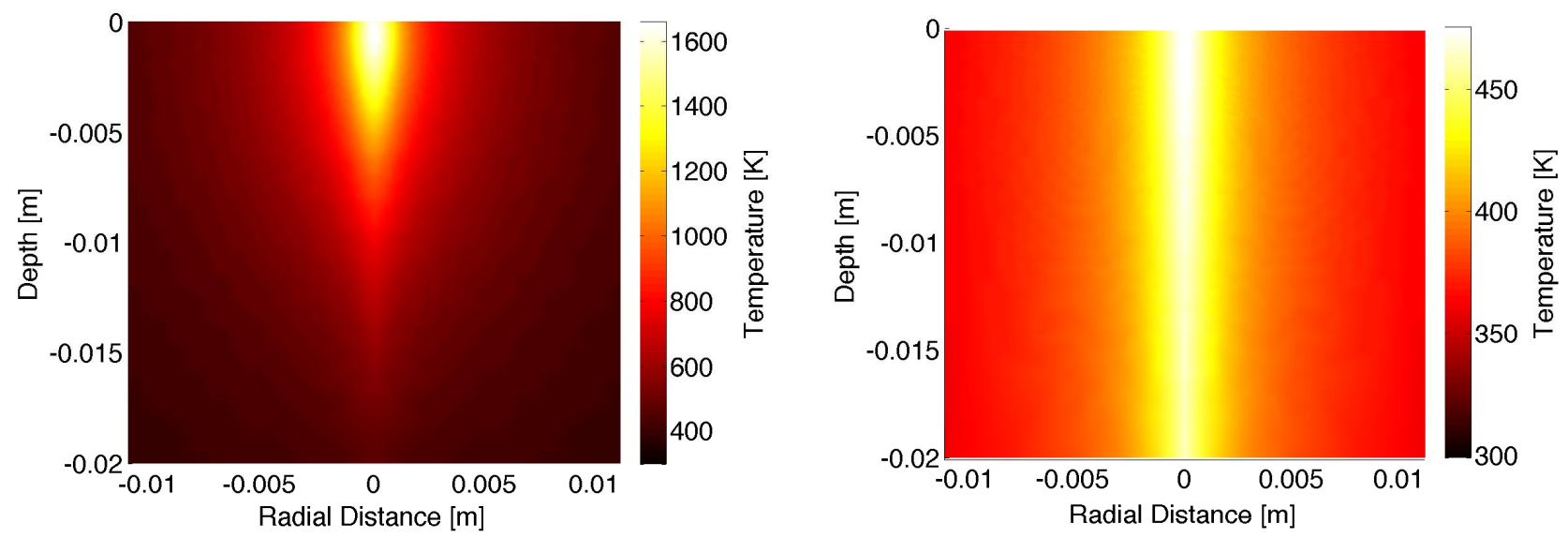

Figure 11. Temperature map inside of the domain for different values of the absorption index $k(\lambda): k(808 n m)=10^{-5}$ (left) and $10^{-6}$ (right). Optical power of $60 \mathrm{~W}$ and spot diameter of $2 \mathrm{~mm}$.

\section{CONCLUSION}

In conclusion, in this paper, a new model of the laser ablation applied to the deflection of an asteroid as been developed. The material properties have been derived from thermochemical tables and thermodynamical considerations. Assuming a sonic ejection of the vaporized matter, the thermal model allows to visualize the temperature profile on the surface and inside the asteroid. By post- processing the results, we can compute the thrust generated in vacuum during laser ablation and assess the power losses during the process. Pending experimental validation, this model will be coupled in the future with laser-based deflection methods and will allow to improve the analysis of these methods. 


\section{ACKNOWLEDGEMENT}

This work is funded by the European Commission's Framework Programme 7, through the Stardust Marie Curie Initial Training Network. Special thanks go to John-Mark Hopkins, David Stothard, John Macarthur, Colin Roberts, Ian Watson and Alison Gibbings for their help with the experimental equipment.

\section{REFERENCES}

[1] Vasile, M., Gibbings, A., Massimo, V., Sanchez, J.-P., Yarnoz, D., Eckersley, S., Wayman, A., Branco, J., Burns, D., Hopkins, J.-M., Colombo, C., and McInnes, C., "Light touch2: Effective solutions to asteroid manipulation. sysnova challenge analysis final report," tech. rep.

[2] Gibbings, A., "A swarm of solar powered lasers for asteroid deflection and exploration," Spaceflight (2012).

[3] Sanchez, J., Colombo, C., Vasile, M., and Radice, G., "Multicriteria comparison among several mitigation strategies for dangerous near earth objects," Journal of Guidance, Control and Dynamics (2009).

[4] Vasile, M., Gibbings, A., Hopkins, J.-M., and Watson, I., "Improved laser ablation model for asteroid deflection," Acta Astronautica (2013).

[5] Kahle, R., Kuhrt, E., Hahn, G., and Knollenberg, J., "Physical limits of solar collectors in deflecting earththreatening asteroids," Aerospace Science and Technology (2006).

[6] NIST-JANAF, "Thermochemical tables."

[7] Ahrens, T. J. and O'Keefe, J. D., "Shock melting and vaporization of lunar rocks and minerals," Earth, Moon, and Planets (1972).

[8] Knight, C., "Theoretical modeling of rapid surface vaporisation with back pressure," AIAA (1979).

[9] Gibbings, A., Laser ablation for the deflection, exploration and exploitation of Near Earth Asteroids, PhD thesis, University of Glasgow (2013).

[10] Robertson, A., "Computation of correlated color temperature and distribution temperature," Journal of the optical society of America (1968).

[11] Anisimov, S. and Lukyanchuk, B., "Selected problems of laser ablation theory.," Physics (2002).

[12] Zeidler, S., Posch, T., Mutschke, H., Richter, H., and Wehrhan, O., "Near-infrared absorption properties of oxygen-rich stardust analogues: The influence of colouring metal ions," Astronomy and Astrophysics . 